\title{
Spectroscopy and Photophysics of Monoazaphenanthrenes III. Luminescence of Phenanthridine and 7,8-benzoquinoline in Crystalline State
}

\author{
M. Norek, B. Kozankiewicz and J. Prochorow \\ Institute of Physics, Polish Academy of Sciences \\ al. Lotników 32/46, 02-668 Warsaw, Poland
}

(Received April 3, 2004)

\begin{abstract}
Fluorescence and phosphorescence spectra and the decay profiles of both these emissions have been investigated for the polycrystals of phenanthridine and 7,8-benzoquinoline, in the liquid helium $(5 \mathrm{~K})$ - room temperature range. These two monoazaderivatives of phenanthrene, which differ only by the position of N-heteroatom in the aromatic ring skeleton of phenanthrene, were found to exhibit very different fluorescence spectra, which also differ greatly in their temperature behavior. Supplementary investigations of the fluorescence of single crystals of 7,8-benzoquinoline have supported classification of observed fluorescence as an excimer fluorescence (caused by the specific arrangement of molecules of 7,8-benzoquinoline in the crystal). In contrary fluorescence of phenanthridine crystals is of the monomeric type. Phosphorescence spectra observed for the crystals of both molecules are very similar, but their temperature dependence is also different. This may be considered as an indication of a different physical mechanism of nonradiative intersystem crossing processes, which are operating between the lowest excited singlet state and the lowest excited triplet state in the crystals of both molecules.
\end{abstract}

PACS numbers: $78.55 . \mathrm{Kz}, 33.50 . \mathrm{Dq}$

\section{Introduction}

In general, substitution of the nitrogen, N-heteroatom(s), into the aromatic skeleton of polycyclic hydrocarbon is connected with introduction of $n$ orbitals (lone pair electrons) and leads to the occurrence of the new $n \rightarrow \pi^{*}$ electronic transitions. This, together with the reduction of the symmetry of the heterocyclic 
molecule, in comparison to its parent polycyclic hydrocarbon, may result in variety of effects which can be induced in absorption and luminescence (fluorescence and phosphorescence) spectra and in nonradiative transitions by different solvents (e.g. hydrocarbon vs. hydroxyllic, or nonpolar vs. polar solvents) - excited $\pi \pi^{*}$ and $n \pi^{*}$ states are usually differently affected by the interaction with solvent molecules and the same is true for the coupling schemes operating within (and between) the singlet and triplet manifolds of excited molecular states (for a review of earlier data see for instance $[1,2])$.

This work is a continuation of our studies of the spectroscopic and photophysical properties of phenanthridine $(\mathrm{PHN})$ and 7,8-benzoquinoline $(\mathrm{BQ})$ - two molecules from the class of monoazasubstituted analogues of phenanthrene, of the structure shown below (with the black dots indicating $\mathrm{N}$ atom position in the aromatic ring skeleton of phenanthrene):

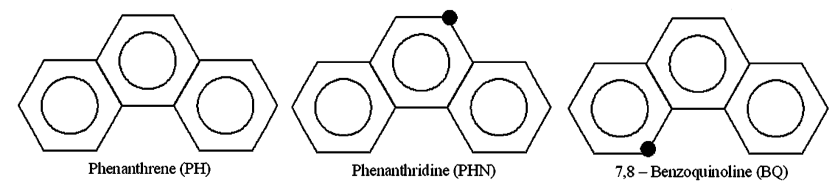

Although these molecules and other members of this family are strong fluorescing agent in protic solvents (serving as fluorescent probes in biological studies) and are of recognized importance in chemistry, life sciences and other related fields, their luminescence and photophysics were studied rather occasionally [3-8], as compared to diaza- or triaza-substituted derivatives of polycyclic hydrocarbons.

The results of our recent investigations of absorption and fluorescence spectra of phenanthridine and 7,8-benzoquinoline in non-polar (and non-hydrogen bonding) solvents, such as $n$-hexane or cyclohexane, and in strongly polar (and hydrogen-bonding) methanol solutions have clearly demonstrated that the first excited, $S_{1}$, singlet state in both molecules is of $\pi \pi^{*}$ character [9]. This conclusion was further confirmed by ab initio analysis of molecular parameters and electronic spectra of both molecules, according to which [10] the lowest excited singlet state of $n \pi^{*}$ character is the second, $S_{2}$, and the third, $S_{3}$, excited state within the singlet states manifold of PHN and BQ, respectively. The observed influence of the solvent on spectral characteristics of absorption and fluorescence spectra of both molecules was rather moderate (with larger Stokes' shift between absorption and fluorescence in the case of BQ molecule upon going from non-polar $n$-hexane to strongly polar methanol), but with a very typical of polycyclic azahydrocarbons effect of the broadening and blurring of vibrational structure of absorption and fluorescence spectra in hydrogen-bonding methanol solution. However, from the photophysical point of view, a very interesting, and probably the most important, result of these investigations was the observation of a much larger (by one order of magnitude) increase in the fluorescence intensity for PHN than for BQ, upon going from $n$-hexane to methanol solution. With estimations of the changes of natural 
radiative rate constants from absorption spectra and measured decay times of fluorescence in both solvents, we arrived at the conclusion that this effect is primarily due to the fact that the rate constant for nonradiative transitions from the first excited $\left(S_{1}\right)$ singlet state is decreased in methanol solution much more for PHN molecule than for BQ molecule. However, we could not point out whether this is to be connected to prevailing changes of rate constants of either an $S_{1} \leadsto S_{0}$ internal conversion, or a $S_{1} \leadsto T_{1}$ intersystem crossing. Differentiation between these two nonradiative channels was virtually impossible, due to the fact that in the case of both molecules under consideration phosphorescence emission is neither observed in $n$-hexane, nor in methanol liquid solutions at room temperature (though it can be observed for some other aza-derivatives of polycyclic hydrocarbons with the lowest excited singlet state of $n \pi^{*}$ character [11-14]).

In order to get a deeper insight into the mechanism of nonradiative transitions from the lowest excited singlet state of PHN and BQ, one has either to study the temperature dependence of fluorescence and its spectral and temporal characteristics in liquid solution, or to look for phosphorescence and its temperature dependence in rigid media, such as crystalline phase, low-temperature frozen solutions or low-temperature isolating matrices of noble gases. Such a combined research effort should lead eventually to understanding the differences in nonradiative relaxation pathway and possible coupling schemes (spin-orbit and/or vibronic spin-orbit) between the singlet and triplet states manifolds of excited PHN and BQ molecules and their relation to different position of nitrogen atom in the parent aromatic ring skeleton of phenanthrene. As the first part of such combined task, we present in this paper the results of investigations of luminescence spectra of phenanthridine and 7,8-benzoquinoline in the crystal state in the temperature range from $5 \mathrm{~K}$ to room temperature.

\section{Experimental section}

Phenanthridine (purum for fluorescence) and 7,8-benzoquinoline (purum 99\%, NT) from Fluka, were recrystallized from 1:9 ethanol/water mixture and after this preliminary purification, very tiny (powderlike) crystals were obtained by sublimation under vacuum.

All experimental studies, with the use of different spectroscopic techniques, were performed with a liquid helium optical cryostat, which allows a control of the temperature within the whole 5-300 $\mathrm{K}$ range.

The fluorescence and phosphorescence spectra (and phosphorescence decays) were investigated with excitation, either with the use of a Lambda Physik FL 1001 dye laser (operating in the 320-360 $\mathrm{nm}$ range; DMQ dye) pumped by a Lambda Physik LPX 100 excimer laser (XeCl, $308 \mathrm{~nm}$ line), or by $308 \mathrm{~nm}$ line of an excimer laser. The emission was observed at a right angle with respect to the excitation. Spectra were resolved with a McPherson 207 monochromator equipped with an EMI 9659 photomultiplier. 
Fluorescence spectra were monitored by the sampling technique, using a Stanford Research SR250 boxcar averager, an amplitude-to-frequency converter and a LightScan PC pulse counter. Decays of fluorescence were measured with the use of the time-correlated single-photon counting technique. The samples were excited by light pulses of $20 \mathrm{ps}$ width, with a $3.8 \mathrm{MHz}$ repetition rate (and of $300 \mathrm{~nm}$ wavelength) obtained as a second harmonic of light emitted by a Coherent 700 dye laser pumped by a mode-locked Antares 76-YAG laser. The setup for these measurements was composed of an avalanche photodiode, a Hammamatsu R28090-07 microchannel plate photomultiplier, a Tennelec TC 454 quad constant fraction discriminator, a Tennelec TC 864 time-to-amplitude converter, and a Nucleus PCA-II multichannel analyser card inserted into PC. Time resolution of this setup was of $c a .50 \mathrm{ps}$.

Phosphorescence spectra were monitored by the photon counting technique with a LightScan PC counter. The prompt emission (fluorescence) following the excitation was filtered out by the synchronized operation of two disc-choppers. Decays of phosphorescence were cumulated with a Stanford Research SR 430 multichannel scaler.

\section{Experimental results and discussion}

At a temperature of $5 \mathrm{~K}$ luminescence spectra of polycrystalline phenanthridine and 7,8-benzoquinoline spread over the same spectral region, from $\sim 28500$ to $\sim 17500 \mathrm{~cm}^{-1}$ (i.e. from $\sim 351$ to $\sim 575 \mathrm{~nm}$ ). As illustrated in Fig. 1 , in both

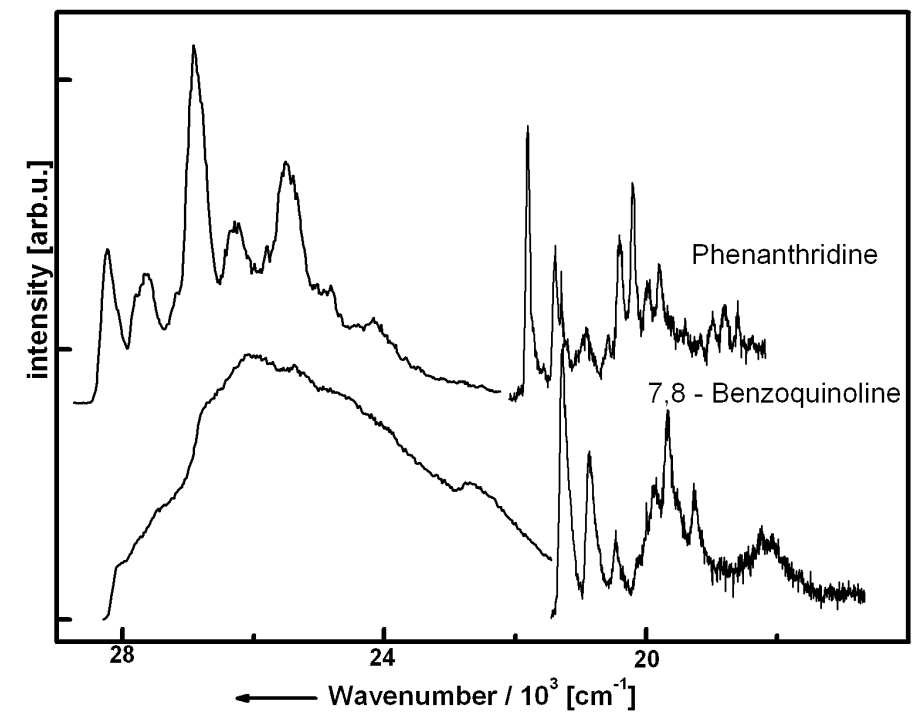

Fig. 1. Fluorescence and phosphorescence spectra, at $5 \mathrm{~K}$, of phenanthridine and 7,8-benzoquinoline crystals excited with $32467 \mathrm{~cm}^{-1}$ energy (308 $\mathrm{nm}$ line of XeCl excimer laser). 
cases the total luminescence spectrum consists of fluorescence and phosphorescence spectra.

An easily noticeable and strikingly distinct difference between the spectra of both molecules, at this temperature, is an apparent lack of vibrational structure in fluorescence spectrum of 7,8-benzoquinoline in comparison to relatively well-structured fluorescence spectrum of phenanthridine. As we will see later on, also the temperature behavior of fluorescence spectra of BQ and PHN molecules is quite different.

\subsection{Fluorescence spectra and their temperature dependence}

Fluorescence of phenanthridine crystals is observed as a relatively intense emission in the range of $28300-22000 \mathrm{~cm}^{-1}$ with only a slightly more developed vibrational structure than the structure of fluorescence spectrum in non-polar hydrocarbon solvents (e.g. $n$-hexane or cyclohexane) at room temperature [9]. Within the error limit, the fluorescence spectrum is independent of the wavelength of excitation.

As it comes from Fig. 1, the fluorescence spectrum of phenanthridine starts with $(0,0)$ origin band located at $28228 \mathrm{~cm}^{-1}$, and is followed by several, broad vibronic bands (with the most intense one at $26914 \mathrm{~cm}^{-1}$ ) and each of these bands has an additional structure. Main features of the vibrational structure are relatively distinct on the high-energy side of the spectrum and are getting less pronounced on the low-energy side and fade away below $24000 \mathrm{~cm}^{-1}$. The observed vibrational frequencies in the fluorescence spectrum of PHN are collected in Table I. The attempt of tentative assignment of the observed frequencies is based

TABLE I

Main vibronic bands of fluorescence spectrum of phenanthridine polycrystals at low temperature $(5 \mathrm{~K})$.

\begin{tabular}{c|c|c|c}
\hline \hline $\begin{array}{c}\text { Energy } \\
\nu\left(\mathrm{cm}^{-1}\right)\end{array}$ & $\Delta \nu\left(\mathrm{cm}^{-1}\right)$ & Intensity & Assignment/Comments* \\
\hline $28228-\nu]$ & $I_{\nu} / I_{(0,0)}$ & \\
27606 & 622 & 1 & $(0,0)$, vibronic origin of fluorescence \\
26914 & 1314 & 2.31 & 616 \\
26292 & 1936 & 1.17 & 1346 \\
25514 & 2714 & 1.56 & $616+1346$ \\
24823 & 3405 & 0.76 & $2 \times 1346$ \\
24166 & 4062 & 0.55 & $3 \times 1346$ \\
\hline
\end{tabular}

${ }^{*}$ Preliminary assignment based on comparison with experimental observations from ground-state IR spectra of phenanthridine isolated in low-temperature $(10 \mathrm{~K})$ argon matrix [15]. 
on the results of our measurements of the ground-state infrared spectra of PHN isolated in the low-temperature $(10 \mathrm{~K})$ argon matrix [15]. However, decreasing intensity and increasing broadness of vibrational bands, on going toward lower energies, very strongly limit the certainty of the values of vibrational frequencies retrieved from the observed fluorescence spectrum.

With the increase in temperature (see Fig. 2), the intensity of fluorescence of PHN is rapidly decreasing - at $20 \mathrm{~K}$, it equals to $c a .0 .6$, and at $40 \mathrm{~K}$ - to 0.4 of the value of integral intensity at $5 \mathrm{~K}$. The decrease in intensity of fluorescence band is connected with blurring of the vibrational features and also with a very clear change in the relative distribution of intensity among different vibronic bands. With an increase in temperature, the high-energy part of the fluorescence spectrum is losing its intensity faster than the low-energy part of the spectrum. At $40 \mathrm{~K}$, the $(0,0)$ band can only be recognized as a shoulder, and the most intense (at $5 \mathrm{~K}$ ) band at $26914 \mathrm{~cm}^{-1}$ is no longer dominating in the spectrum. At $80 \mathrm{~K}$, fluorescence is only slightly stronger than the background noise and above this temperature can no longer be observed (or reliably measured).

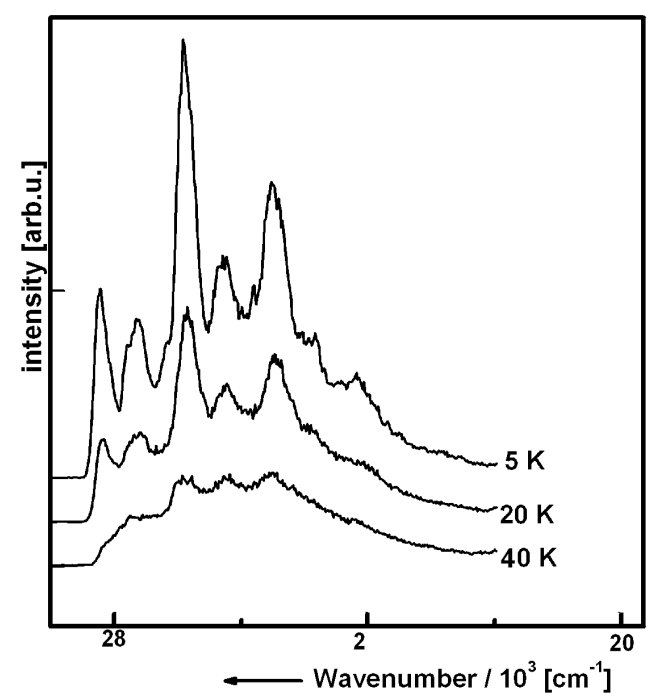

Fig. 2. Fluorescence spectra of phenanthridine crystals at different temperatures (as indicated). Excitation energy $32467 \mathrm{~cm}^{-1}$.

Temperature changes of the intensity are accompanied by the changes of the decay times of fluorescence. The decay of fluorescence at $5 \mathrm{~K}$, monitored at the origin $(0,0)$ band, is monoexponential with the decay time of $12.3 \mathrm{~ns}$. For the most intense band at $26914 \mathrm{~cm}^{-1}$ the observed decay is also monoexponential but its decay time of $11.7 \mathrm{~ns}$ is slightly shorter (we should mention, however, that for all temperatures above $5 \mathrm{~K}$, the observed decay profiles are double exponential with the short component of the decay of $3.4-2.4 \mathrm{~ns}$ ). The decay times of fluo- 
TABLE II

Temperature changes of the decay times of fluorescence*, $\tau$, of phenanthridine (PHN) and 7,8-benzoquinoline (BQ).

\begin{tabular}{c|c|c}
\hline \hline Temp. $[\mathrm{K}]$ & PHN $\tau[\mathrm{ns}]$ & BQ $\tau[\mathrm{ns}]$ \\
\hline 5 & 11.7 & 15.8 \\
20 & 10.4 & 15.9 \\
40 & 9.2 & 15.5 \\
60 & 6.7 & 14.9 \\
80 & 6.1 & 13.5 \\
100 & - & 11.9 \\
140 & - & 9.7 \\
200 & - & 7.8 \\
250 & - & 7.0 \\
300 & - & 6.4 \\
\hline *Decays monitored at $26914 \mathrm{~cm}^{-1}$ \\
for PHN and at 26000 $\mathrm{cm}^{-1}$ for BQ \\
crystal.
\end{tabular}

rescence for different temperatures (all measured for the band at $26914 \mathrm{~cm}^{-1}$ ) are collected in Table II. We note that the observed shortening of the decay times, in the temperature range accessible for their measurements, is relatively moderate in comparison to the changes of intensity of fluorescence in the same range of temperatures (i.e. 5-80 K).

Temperature changes of fluorescence of polycrystalline PHN, connected with a continuous change of the relative distribution of intensity toward lower energies (on the expense of the high-energy part of the spectrum) with increasing temperature, suggest that the kinetics of fluorescence may be controlled by, very typical for organic molecular crystals, trapping processes of excitation energy [16]. It looks like the excitation energy (singlet excitons) is being thermally repumped from shallow to deeper traps located below the bottom of singlet excitonic band. At this point we should mention that temperature changes of the fluorescence spectrum of polycrystalline samples of PHN recall to some extent of temperature changes of fluorescence of chemically mixed crystals, especially those formed with acridine (i.e. monoazaanthracene) serving as a guest in other crystalline matrices [17-19]. However, polycrystalline samples of PHN should probably be considered as more amorphous than single crystals, and other factors (characterizing imperfections in the bulk of tiny crystallites) must be taken into account in the description of observed temperature changes of the fluorescence.

As was mentioned at the beginning of this section, the fluorescence spectrum of polycrystalline 7,8-benzoquinoline observed at $5 \mathrm{~K}$ is a very broad, unsymmet- 
rical band practically lacking any distinct vibrational structure (cf. Fig. 1). There is a shoulder around $28000 \mathrm{~cm}^{-1}$, and some other shoulders are coming before the maximum located at $\sim 26100 \mathrm{~cm}^{-1}$. This is somewhat surprising, especially in view of the fact that in $n$-hexane solution at room temperature, fluorescence spectra of PHN and BQ [9] have a very similar vibrational structure (they differ only in the position of the $0-0$ origin transitions by $246 \mathrm{~cm}^{-1}$ ). Even more surprising is the observed temperature behavior of the fluorescence spectrum of BQ, which is illustrated in Fig. 3. In the temperature range from $5 \mathrm{~K}$ to $\sim 60 \mathrm{~K}$ the intensity

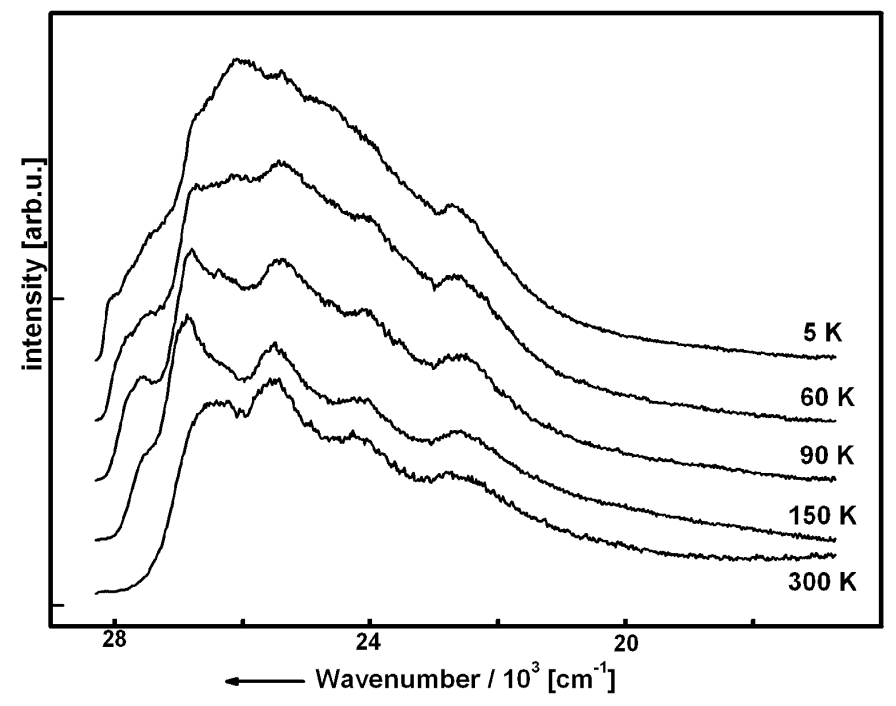

Fig. 3. Fluorescence spectra of 7,8-benzoquinoline crystals at different temperatures (as indicated). Excitation energy $32467 \mathrm{~cm}^{-1}$.

of fluorescence practically does not change (within the error limits of measurements). For higher temperatures (above $60 \mathrm{~K}$ ) the intensity of fluorescence starts to decrease faster, and at the same time the shape of fluorescence band starts to change, disclosing more visible structural features. In the temperature range of 90-150 K, in a very broad fluorescence spectrum at least five maxima of intensity (presumably corresponding to different vibronic transitions) can be picked up at: $27585,26825,25496,24135$, and $22746 \mathrm{~cm}^{-1}$ (with $\pm 20 \mathrm{~cm}^{-1}$ accuracy). It is also clearly seen that at elevated temperatures, the fluorescence spectrum of BQ undergoes changes in the same manner as that observed for fluorescence of PHN. It is, a high-energy part of the fluorescence spectrum is continuously losing its intensity (e.g. the maximum of $27585 \mathrm{~cm}^{-1}$, which is the highest energy maximum at $90 \mathrm{~K}$, is seen as a shoulder at $150 \mathrm{~K}$, and at $300 \mathrm{~K}$ cannot be traced at all). Altogether, the net result of the temperature changes of fluorescence between the lowest $(5 \mathrm{~K})$ and the highest $(300 \mathrm{~K})$ temperature is seen as a (apparent) shift of the fluorescence spectrum toward lower energies - at $300 \mathrm{~K}$, the most intense 
maximum is the one at $25496 \mathrm{~cm}^{-1}$. The decays of BQ fluorescence (monitored for the center of fluorescence band, at $26000 \mathrm{~cm}^{-1}$ ) are always monoexponential with the decay time decreasing much slower than in the case of PHN fluorescence (cf. Table II).

A broad and diffused fluorescence band, nearly lacking any vibrational structure at deep temperatures, and with a more distinct structure developing within the limited range of elevated temperatures (90-150 K), rises a question about the compositions and the nature of polycrystalline samples of BQ, which are being investigated. To our best knowledge, very little is known about the crystals of monoazaphenanthrenes. The crystal structure and the molecular structure of PHN have been determined [20], and subsequently redetermined [21] by X-ray diffraction. On the other hand, no crystallographic data concerning 7,8-benzoquinoline are available.

The character of observed temperature changes of the fluorescence of polycrystalline BQ seems to suggest that we may deal with the samples composed of different crystalline modifications of BQ, which may be converted the one into the other upon heating. The best known example of such polymorphism among N-heterocyclic hydrocarbons, is the case of acridine (monoazaanthracene) - Nheterocyclic analogue of anthracene, with nitrogen atom in the central ring of aromatic skeleton of anthracene. Five different polymorphs of acridine have been identified and characterized, also with respect to their luminescence [22-25], and the important thing is that each of these five crystalline modifications of acridine has a different fluorescence spectrum. This may be an indication that in the case of tiny (powderlike) crystallites grown in this work by sublimation method, our polycrystalline samples of BQ may be composed of different polymorphs, with different fluorescence spectra dominating in different temperature ranges.

On the other hand, the observed fluorescence spectrum bears a very close resemblance to the excimeric fluorescence. In the case of mentioned just above acridine, in the crystal structure of acridine II and acridine III, two, among its five identified and structurally characterized crystalline modifications [26, 27], molecules are arranged in parallel pairs, which provides conditions necessary for occurrence of excimers and excimer fluorescence in crystalline state [28, 29]. Namely, in acridine II (monoclinic, with 8 molecules in the unit cell and the space group $P 2_{1} / a$ ) with two molecules per asymmetric unit, an arrangement of molecules is quite complex. In the packing units (each containing two molecules) the molecules are arranged in two types of antiparallel pairs about the center of symmetry (A and B pairs). In orientation $A$, in normal projection molecules are not exactly overlapped but are staggered. In orientation B, with a larger distance between molecules, in a normal projection there is only an overlap between terminal aromatic rings of the two acridine molecules [27]. The observed fluorescence spectrum of acridine II is a very broad unsymmetrical band, which is considered as a mixture of molecular (monomeric) emission that originates from B molecules and excimeric emission 
from molecular pairs B. Such characteristics of fluorescence spectrum seem to fit the presented above fluorescence spectrum of $\mathrm{BQ}$ at deep temperatures and this may suggest that instead of mixture of polymorps we may have in polycrystalline sample only one type of crystallites with the pairwise structure of molecules. We will return to this issue in Sec. 3.3. with the preliminary report of investigations of fluorescence of the single crystal of BQ at $5 \mathrm{~K}$.

In a summary of the above-described results of observations of fluorescence of PHN and BQ polycrystals, we must stress the most remarkable differences between the temperature behavior of their fluorescence spectra, and these are: (a) intensity of fluorescence of BQ crystals is changing very moderately with temperature - even at $300 \mathrm{~K}$ fluorescence of $\mathrm{BQ}$ is still observed and measurable, while fluorescence of PHN crystals cannot be observed above $80 \mathrm{~K}$; (b) the decays of BQ fluorescence are always monoexponential, with the decay time decreasing much slower than in the case of PHN fluorescence (cf. Table II). Such differences in the temperature behavior of the intensity and temporal parameters of fluorescence for PHN and BQ crystals, are probably connected with the different schemes of the spin-orbit (and/or spin-orbit vibronic) coupling operating between the manifolds of excited singlet and triplet states of both molecules, which should be reflected also in the spectral and temporal characteristics of their phosphorescence.

\subsection{Phosphorescence spectra and their temperature dependence}

Phosphorescence spectrum of PHN crystals (cf. Fig. 1), at $5 \mathrm{~K}$, starts with relatively sharp $(0,0)$ origin band located on the far tail of fluorescence at $21812 \mathrm{~cm}^{-1}$, and extends down to the red region (of $\sim 17000 \mathrm{~cm}^{-1}$ ). A separation between the $(0,0)$ origin transitions in fluorescence and phosphorescence spectra, which approximates the separation between the lowest excited singlet and triplet state $\Delta\left(S_{1}-T_{1}\right)$ equals $6416 \mathrm{~cm}^{-1}$.

The vibrational structure of phosphorescence spectrum is relatively sharp and practically each vibrational band shows an additional "fine" structure (cf. inset to Fig. 4). Frequencies of the most distinct vibronic bands* are collected in Table III, which also contains the vibrational frequencies observed in the ground-state IR spectrum of PHN isolated in low-temperature $(10 \mathrm{~K})$ argon matrix [15]. A comparison of both sets of the data in Table III shows quite a good correspondence between the vibrational frequencies present in the phosphorescence spectrum and those found in the IR spectrum. We should note also that the sets of vibrational frequencies which facilitate $S_{1} \rightarrow S_{0}$ (fluorescence) and $T_{1} \rightarrow S_{0}$ (phosphorescence) radiative transitions are different (cf. Tables I and III) and that the fluorescence spectrum is built on in-plane vibrational modes, while in

\footnotetext{
${ }^{*}$ A detailed analysis of the vibrational structure of the phosphorescence spectra of PHN and BQ is not carried out in this paper. Such analysis will be presented elsewhere, together with the results of analysis of quasi-linear luminescence spectra (fluorescence and phosphorescence) observed for both molecules isolated in Shpol'skii matrices ( $n$-hexane, at $6 \mathrm{~K}$ ) and in low-temperature $(5 \mathrm{~K})$ neon matrix.
} 


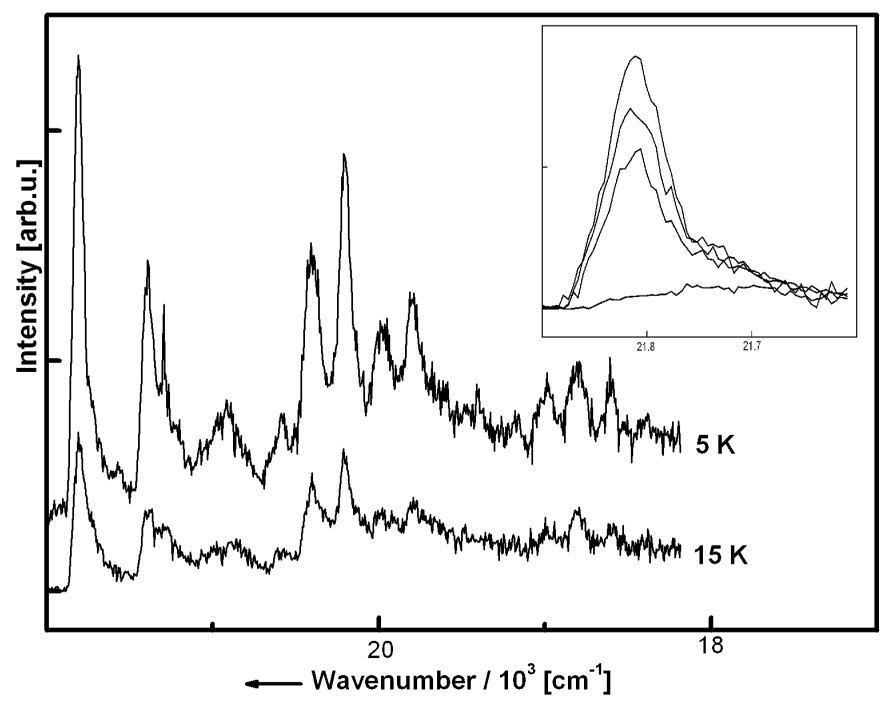

Fig. 4. Phosphorescence spectra of phenanthridine crystals at the temperatures (as indicated). The inset shows a magnified region of the $(0,0)$ origin band $\left(21812 \mathrm{~cm}^{-1}\right)$ of phosphorescence at 5,10,15, and $20 \mathrm{~K}$ (from top to bottom). Excitation energy $32467 \mathrm{~cm}^{-1}$.

the phosphorescence spectrum both, the in-plane and out-of-plane vibrations are engaged.

Temperature changes of the phosphorescence spectrum of PHN are illustrated in Fig. 4. From 5 to $15 \mathrm{~K}$, intensity of phosphorescence is decreasing continuously, but the decrease is moderate and at $15 \mathrm{~K}$ the integral intensity of phosphorescence amounts to $\sim 80 \%$ of that at $5 \mathrm{~K}$. Also the general shape and vibrational structure of the spectrum do not change, and as in the case of fluorescence spectrum some slight redistribution of the relative intensity in favor of low-energy part of the spectrum can be observed. These changes of intensity are accompanied by a smaller relative shortening of the decay time of phosphorescence, which (monitored for $0-0$ transition at $21812 \mathrm{~cm}^{-1}$ ) is: 756,755 , and $702 \mathrm{~ms}$, at 5,10 , and $15 \mathrm{~K}$, respectively. However, an increase in temperature above $15 \mathrm{~K}$ results in dramatic quantitative and qualitative changes of the phosphorescence spectrum. The inset to Fig. 4 illustrates these changes for the $(0,0)$ origin band of the phosphorescence spectrum. It is seen that at $20 \mathrm{~K}$, the origin band, which is the most intense vibrational band of the spectrum in the range of 5-15 K, ceases to exist in a normal sense - the remnants of this band are seen as a very weak (about $10 \%$ of its initial integral intensity at $5 \mathrm{~K}$ ) broad and flat band. The rest of the spectrum is also deprived of its structure and the maximum of flat envelope of the phosphorescence, only slightly exceeding the background noise, can be located at $c a .19200 \pm 200 \mathrm{~cm}^{-1}$. We notice that the decay time of phosphorescence at $20 \mathrm{~K}$ (measured at $27000 \mathrm{~cm}^{-1}$ position) equals $c a .420 \mathrm{~ms}$. It seems obvious that 
TABLE III

Main vibronic bands of phosphorescence spectrum of phenanthridine polycrystals at low temperature $(5 \mathrm{~K})$.

\begin{tabular}{c|c|c|c}
\hline \hline $\begin{array}{c}\text { Energy } \\
\nu\left(\mathrm{cm}^{-1}\right)\end{array}$ & $\Delta \nu\left(\mathrm{cm}^{-1}\right)$ & Intensity & Assignment/Comments* \\
\hline 21812 & 0 & 1 & $(0,0)$, vibronic origin of phosphorescence \\
21394 & 418 & 0.57 & 429 \\
20916 & 896 & 0.27 & 894 \\
20584 & 1228 & 0.24 & 1242 \\
20367 & 1445 & 0.55 & 1453 \\
20212 & 1600 & 0.79 & 1596 \\
19990 & 1822 & 0.44 & \\
19814 & 1998 & 0.50 & \\
19396 & 2416 & 0.29 & \\
18988 & 2824 & 0.33 & \\
18808 & 3004 & 0.35 & \\
18591 & 3221 & 0.32 & \\
18374 & 3438 & 0.24 & \\
\hline
\end{tabular}

*Preliminary assignment based on comparison with experimental observations from ground-state IR spectra of phenanthridine isolated in lowtemperature $(10 \mathrm{~K})$ argon matrix [15].

at this temperature the observed emission comes from the emitting species which are different than those populated at low temperatures (in 5-15 K temperature range). If these species are the triplet excitons traps, then their depth (location below the bottom of the triplet excitonic band) is quite large.

The general outlook of the phosphorescence spectrum of polycrystalline BQ at a temperature of $5 \mathrm{~K}$ is very similar to the phosphorescence spectrum of $\mathrm{PHN}$ (cf. Figs. 1, 4 and 5). The most intense and relatively sharp $(0,0)$ origin band is located at $21275 \mathrm{~cm}^{-1}$, and is shifted by $537 \mathrm{~cm}^{-1}$ toward lower energies as compared to the position of $(0,0)$ band in the case of phosphorescence spectrum of PHN. A separation between the (0-0) origin transitions in fluorescence and phosphorescence spectra, which approximates the separation between the lowest excited singlet and triplet cannot be determined in this case, as the (0-0) origin transitions in fluorescence spectrum at $5 \mathrm{~K}$ could not be observed. If we treat a maximum, revealed at $27585 \mathrm{~cm}^{-1}$ (in the spectrum at $120 \mathrm{~K}$ ) as the $(0,0)$ origin band of fluorescence, then the lower limit for the $\Delta\left(S_{1}-T_{1}\right)$ separation would be set as $6310 \mathrm{~cm}^{-1}$ and this value is comparable to the $\Delta\left(S_{1}-T_{1}\right)$ separation in the case of PHN. However in view of the earlier discussion of possible polymorphism of crystalline samples of BQ such evaluation may not be well justified. Other vibrational bands which are present in the phosphorescence spectrum of BQ are 


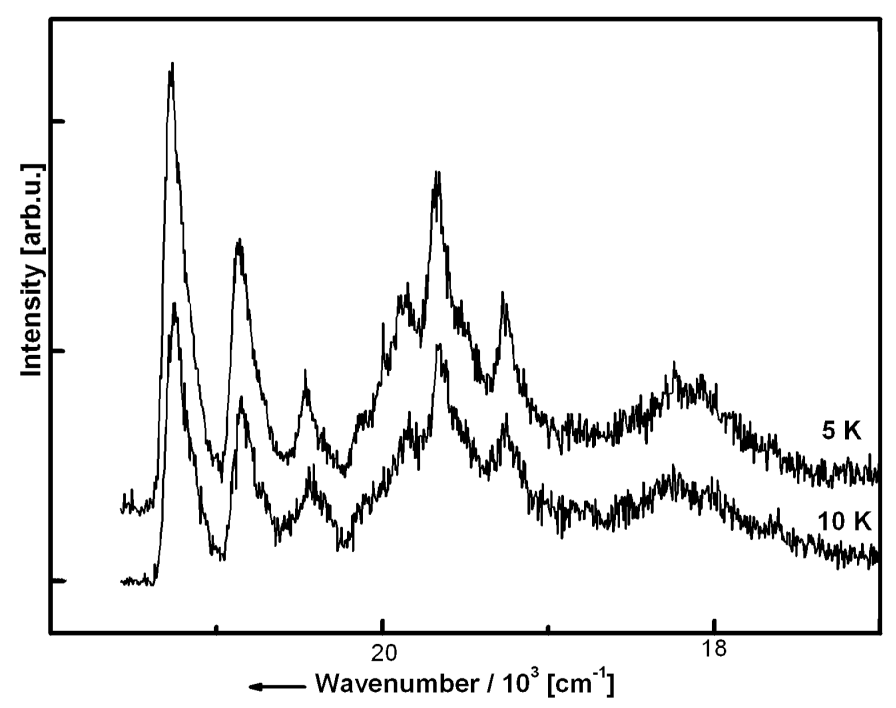

Fig. 5. Phosphorescence spectra of 7,8-benzoquinoline crystals at different temperatures (as indicated). Excitation energy $32467 \mathrm{~cm}^{-1}$.

TABLE IV

Main vibronic bands of phosphorescence spectrum of 7,8-benzoquinoline polycrystals at a temperature of $5 \mathrm{~K}$.

\begin{tabular}{c|c|c|c}
\hline \hline $\begin{array}{c}\text { Energy } \\
\nu\left(\mathrm{cm}^{-1}\right)\end{array}$ & $\Delta \nu\left(\mathrm{cm}^{-1}\right)$ & Intensity & Assignment/Comments* \\
\hline $21275-\nu]$ & $I_{\nu} / I_{(0,0)}$ & \\
\hline 20868 & 0 & 1 & $(0,0)$, vibronic origin of phosphorescence \\
20454 & 807 & 0.61 & (region not available in [30]) \\
19892 & 1383 & 0.48 & 821.1 \\
19664 & 1611 & 0.70 & 1388.9 \\
19277 & 1998 & 0.49 & 1603.1 \\
18240 & 3035 & 0.34 & \\
\hline
\end{tabular}

* Preliminary assignment based on comparison with data of ground-state mid-IR spectra of 7,8-benzoquinoline isolated in low-temperature $(15 \mathrm{~K})$ argon matrix [30].

collected in Table IV, and they seem to fit the frequencies observed in the mid-IR spectrum of 7,8-benzoquinoline isolated, at $15 \mathrm{~K}$, in argon matrix [30].

Let us draw attention to the fact that although fluorescence spectrum of $\mathrm{BQ}$ is practically unstructured and very broad "excimer-like" band, its phosphorescence spectrum has a very well-developed structure (much alike as in the case of PHN phosphorescence), apparently with no relations to the excimeric emission. Exactly the same kind of fluorescence and phosphorescence spectra were observed 
in the case of acridine II crystal - a broad and structureless fluorescence spectrum, and a very rich and sharp structure of phosphorescence spectrum. Investigations of phosphorescence and its structure, at deep temperatures $(1.7 \mathrm{~K})$ and under high resolution conditions, have definitely ruled out triplet excimers as a way for dissipation of singlet excitons [31].

If we compare changes of the phosphorescence spectra of BQ and PHN with the rise of temperature, then we see that in the temperature range of 5-10 K changes of BQ phosphorescence are paralleling those observed for PHN (cf. Fig. 5 and Fig. 4) - the intensity change is very moderate and also the general shape and vibrational structure of the spectrum practically do not change. However, for the temperatures higher than $10 \mathrm{~K}$ the drop of the intensity is drastic and the phosphorescence of $\mathrm{BQ}$ can no longer be measured (while phosphorescence of PHN is still fairly intense at $15 \mathrm{~K}$ and can be traced up to $\sim 20 \mathrm{~K}$ ).

The decay times of $\mathrm{BQ}$ phosphorescence (measured for $(0,0)$ band of $21275 \mathrm{~cm}^{-1}$ ) are equal to $1.40 \mathrm{~s}$ and $1.05 \mathrm{~s}$ at $5 \mathrm{~K}$ and $10 \mathrm{~K}$, respectively, and thus are approximately twice as long as the decay times of PHN phosphorescence at the same temperatures. At higher temperatures (above $10 \mathrm{~K}$ ) the intensity of phosphorescence is too weak for the decays to be monitored.

It is clear that the nonradiative relaxation of the lowest excited, $T_{1}$, triplet state of BQ crystals is more efficient than the one for PHN crystals. This fact could be related, in the first approximation, to a smaller $T_{1}-S_{0}$ energy gap in the case of BQ (by $c a .500 \mathrm{~cm}^{-1}$ ) as compared to the case of PHN (cf. data in Tables III and IV). We must, however, bear in mind that in crystal phase differences in the relaxation of excited triplet are controlled and governed, to a large extent, by the depth and distributions of the traps for triplet excitons, below the triplet excitonic band, which may be very specific for each of both, the PHN and BQ crystals.

\subsection{Fluorescence spectrum of 7,8-benzoquinoline single crystal at the temperature of $5 \mathrm{~K}$}

All reservations, concerning the composition of polycrystalline samples and/or possible specific crystal structure of 7,8-benzoquinoline, which were discussed in Sec. 3.1, as well as the reported above experimental findings, are clearly indicating that the investigations of neat single crystals of PHN and BQ should be carried out. This would take care of additional effects (specific for imperfections in the bulk of polycrystalline samples) and would enable a more transparent interpretation of the results of observations.

Without switching to other methods of crystal growing, we were successful in growing, by slow vacuum sublimation, the needle-like, pale yellow (almost transparent) BQ single crystals, large enough for spectroscopic (and cryogenic) investigations ( $\sim 5 \mathrm{~mm}$ long). And although their crystal structures have not been yet determined, we want to show here their fluorescence spectrum for a preliminary comparison with the fluorescence of polycrystalline samples of BQ. Such a 


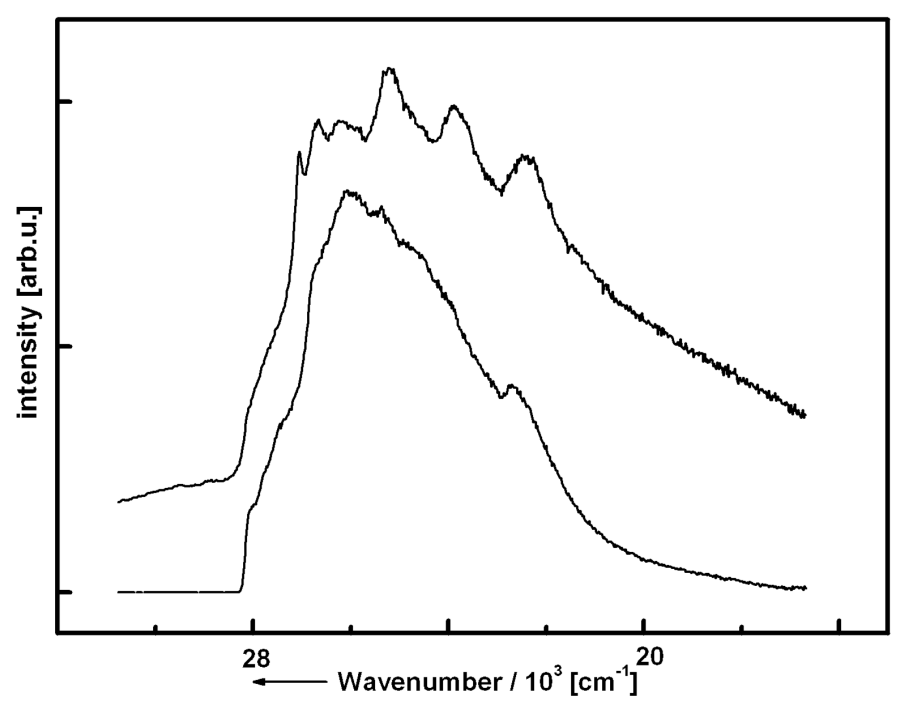

Fig. 6. Phosphorescence spectra, at $5 \mathrm{~K}$, of 7,8-benzoquinoline single crystal (top) and polycrystals (bottom). Excitation energy $32467 \mathrm{~cm}^{-1}$.

comparison is given in Fig. 6, which illustrates the fluorescence spectrum of single crystal of $\mathrm{BQ}$ (at a temperature of $5 \mathrm{~K}$ ) and that observed for polycrystalline samples of BQ registered at the same temperature (cf. also Figs. 1 and 3). As it is seen, a very broad envelop of the fluorescence band of the single crystal (at $5 \mathrm{~K}$ ) has a much better developed structure and a much more intense low-energy part than in the case of fluorescence band of its polycrystals. As a matter of fact, the fluorescence of single crystal observed at $5 \mathrm{~K}$ is in very close resemblance with the spectra of polycrystals in the $90-150 \mathrm{~K}$ temperatures range (cf. Fig. 3), with the most intense maxima $\left(25218,23893\right.$, and $22489 \mathrm{~cm}^{-1}$ ) shifted to the red (to lower energies) by $c a .250 \mathrm{~cm}^{-1}$ as compared to spectra of polycrystals. However, in this simple comparison we have omitted the very fact that the fluorescence band of single crystal of BQ is almost twice as wide as that of polycrystals. We mention also that the temperature changes of single crystal fluorescence are very moderate, with the effect of the decrease in intensity of high-energy part of the spectrum and simultaneous increase in its low-energy part (known from the observations of polycrystals).

We believe that the main message which comes from the comparison of the fluorescence spectra of single crystal and polycrystals of BQ is ruling out the supposition about the mixture of structural (polymorphic) modifications of $\mathrm{BQ}$ in the polycrystalline samples. It seems to point out that $\mathrm{BQ}$ crystallites (as well as its single crystal), which are grown by vacuum sublimation, are of complex pairwise arrangement of the molecules in the crystal lattice which results in resonance interaction leading to the excimeric fluorescence emission. 


\section{Summary and closing remarks}

Our investigations of spectral and temporal characteristics of the luminescence emission (fluorescence and phosphorescence ) of polycrystals of PHN and $\mathrm{BQ}$ at a low temperature of $5 \mathrm{~K}$ as well as their temperature dependence have revealed some remarkable differences between these molecules in the crystalline state, which can be summarized as follows:

- First of all, there is a qualitative difference between their fluorescence spectra. PHN crystals exhibit a regular, monomeric-type fluorescence, while the fluorescence of BQ crystals is of typical excimeric fluorescence. This seems to be inherently connected with different packing and arrangement of the molecules in the crystal lattice of each of both molecules.

- Different nature and type of the fluorescence emission result in the remarkably different temperature range accessible for its observations. And thus, the fluorescence of PHN crystals is observed (and measurable) in a temperature range of 5-80 K, while the range for the BQ fluorescence is 5-300 K (and surely higher).

- Phosphorescence spectra are very similar for both crystals. They are well structured and their vibrational features can be identified in the available ground-state IR spectra of molecules isolated in low-temperature argon matrices.

- As in the case of fluorescence emission, also phosphorescence displays differences in the temperature behavior; a temperature range accessible for observation of the phosphorescence is $5 \mathrm{~K}$ to $\sim 20 \mathrm{~K}$ for PHN crystals, and only $5 \mathrm{~K}$ to $10 \mathrm{~K}$ for $\mathrm{BQ}$ crystals. In view of a rather small difference in the energy of the $T_{1}$ triplet state in both crystals $\left(c a .500 \mathrm{~cm}^{-1}\right)$ and with a much slower depopulation of the first excited, $S_{1}$, singlet state of BQ crystal upon increasing temperature, this would mean that the mechanisms of the nonradiative, $S_{1} \leadsto T_{1}$, intersystem crossing in BQ crystals must be different from those operating in the case of PHN crystals. Such differences must be connected with a different pathway for relaxation of presumable excimer state (which decays to monomer triplet state) in BQ crystal.

In our opinion experimental results presented in this work are a very clear demonstration of how a seemingly secondary difference in the position of $\mathrm{N}$ atom, in the aromatic ring skeleton of parent phenanthrene molecule, may deliver substantial differences of the emission properties in the crystalline state of monoazaphenanthrenes. However, in order to proceed along that line, one must define the crystallographic structure of $\mathrm{BQ}$, and this is now in course of investigations. 


\section{Acknowledgments}

We gratefully acknowledge a partial support of this work by the State Committee for Scientific Research under project 5 P03B 13620.

\section{References}

[1] R.S. Becker, Theory and Interpretation of Fluorescence and Phosphorescence, Wiley Interscience, New York 1969.

[2] E.C. Lim, in: Excited States, Ed. E.C. Lim, Vol. 3, Academic Press, New York 1977, p. 305.

[3] M. Nakamizo, Spectrochim. Acta 22, 2039 (1966).

[4] A. Grabowska, B. Pakuła, J. Panciř, Photochem. Photobiol. 10, 615 (1969).

[5] I. Janic, A. Kawski, Bull. Acad. Pol. Sci, Ser. Sci. Math. Astr. Phys. XX, 235 (1972).

[6] I. Janic, A. Kawski, Bull. Acad. Pol. Sci, Ser. Sci. Math. Astr. Phys. XX, 519 (1972).

[7] C.J. Marzzacco, G. Deckey, R. Colarulli, G. Siuzdak, A.M. Halpern, J. Phys. Chem. 93, 2935 (1989).

[8] T.G. Pavlopoulos, J. Photochem. Photobiol. A, Chemistry 149, 45 (2002).

[9] M. Norek, J. Dresner, J. Prochorow, Acta Phys. Pol. A 104, 425 (2003).

[10] I. Deperasińska, J. Prochorow, Acta Phys. Pol. A 104, 601 (2003).

[11] M.A. El-Sayed, J. Chem. Phys. 38, 2834 (1963); 36, 573 (1962).

[12] B. Cohen, H. Baba, L. Goodman, J. Chem. Phys. 43, 2902 (1965).

[13] L. Logan, J. Ross, J. Chem. Phys. 43, 2903 (1965).

[14] B.J. Cohen, L. Goodman, J. Chem. Phys. 46, 714 (1967).

[15] M.J. Nowak, L. Lapiński, M. Norek, I. Deperasińska, to be published.

[16] M. Pope, C.E. Swenberg, Electronic Process in Organic Cystals, Ch. I, Oxford University Press, New York 1982.

[17] O. Morawski, B. Kozankiewicz, J. Prochorow, R. Radomski, Chem. Phys. Lett. 150, 307 (1988).

[18] O. Morawski, B. Kozankiewicz, J. Prochorow, R. Radomski, Chem. Phys. Lett. 173, 412 (1990).

[19] O. Morawski, J. Prochorow, Chem. Phys. Lett. 242, 253 (1995).

[20] P. Roychowdhury, Acta Crystallogr. B 29, 1362 (1973).

[21] W.A. Brett, P. Rademacher, R. Bose, Acta Crystallogr. C 49, 1564 (1993).

[22] B.P. Clarke, J.M. Thomas, J.O. Williams, Chem. Phys. Lett. 35, 251 (1975).

[23] J.O. Williams, B.P. Clarke, M. J. Shaw, Chem. Phys. Lett. 39, 142 (1976).

[24] J.O. Williams, B.P. Clarke, J. Chem. Soc. Faraday Trans. II 73, 514 (1977).

[25] O. Morawski, J. Prochorow, R. Radomski, Mol. Cryst. Liq. Cryst. 230, 173 (1993).

[26] D.C. Phillips, Acta Crystallogr. 9, 237 (1956). 
[27] D.C. Phillips, F.R. Ahmed, W.H. Barnes, Acta Crystallogr. 13, 365 (1960).

[28] M.D. Cohen, Z. Ludmer, V. Yakhot, Phys. Status Solidi 67, 51 (1975).

[29] R. Cohen, Z. Ludmer, V. Yakhot, Chem. Phys. Lett. 35, 271 (1975).

[30] A.I. Mattioda, D.M. Hudgins, C.W. Bauschlicher, Jr., M. Rosi, L.J. Allamandola, J. Phys. Chem. A 107, 1486 (2003).

[31] O. Morawski, J. Prochorow, Acta Phys. Pol. A 88, 469 (1995). 